\title{
The Importance of Data in an Exclusive Human Milk Diet: Key Concepts and Points of Consideration
}

Mitchell Goldstein, MD, MBA, CML

\section{"Data is a term that is not hard to define but is difficult to describe. We recognize data for what it is but fail to understand how to interpret it. In the interest of qualifying data and preserving effort, more rigorous challenges to data are often put aside to qualify or validate the effort."}

Data is a term that is not hard to define but is difficult to describe. We recognize data for what it is but fail to understand how to interpret it. In the interest of qualifying data and preserving effort, more rigorous challenges to data are often put aside to qualify or validate the effort. Such is the case with the study of human milk. Although a study could be found to support just about any conclusion one is seeking, when reviewed in toto an exclusive human milk diet (EHMD) defined as mother's milk and/or pasteurized donor milk plus a human milk-based fortifier has proved to be best for babies time and time again. (1-5) Not all donor milk is created equal, however, and relying on pasteurized donor milk studies to draw conclusions about donor milk sterilized by other means is not appropriate. Any inference that pasteurization is a form of sterilization is incorrect. (6)

\section{The importance of appropriate data analysis}

Although statistics do not really lie, they can be manipulated to mask the truth, especially if the most meaningful benchmark for the data analysis is not applied. For example, setting an appropriate endpoint and studying an appropriate sample size are both critically important.

A power analysis for an appropriate metric should be performed. (7) Whether that metric focuses on increases in lean body mass, improved immunological wellbeing, or reduction in NEC, clinically meaningful differences must be defined. Studying a vast number of points is not a viable solution to this problem but will invariably qualify even the most numerically similar samples as smaller and smaller differences are deemed significant. (8) Yes, quantity is important, but this quantity must be qualified by a study design that supports realistic conclusions.

In Neonatology, the provisioning of an exclusive human milk diet has been demonstrated as superior in numerous trials. Although data exist for various means to improve clinical outcomes, increase growth velocity, and improve other aspects of newborn health, few interventions compare with human milk. (1-5)

\section{When analyzed appropriately, the totality of the data strongly support the use of an EHMD}

To date, roughly fifty studies provide evidence of the overwhelming success of EHMD in providing solid metrics of health quality improvement in an at-risk population defined by higher risks for morbidity and mortality.

The quality and quantity of these data are critically important, as is the sheer preponderance of the evidence, be it different centers or different protocols. If all these studies have similar outcomes, this is not by chance. That being said, studies that look at a range of human milk supplementation or supplementation that varies according to the availability of mom's own milk can be called into question. For there to be equipoise, bias in thought and bias in the sample must be avoided. The statistical methodology must be appropriate for the data. Specifically, it is inappropriate to use multiple T-tests to study the differences between different points of comparison in a study. By chance, certain comparisons may show up positive, despite the lack of true statistical significance. (9) A Bonferroni correction can help provide clarity and adjust for the multiple comparison problem, but other statistical methods may provide a better and more thorough analysis. Post hoc testing can help define where differences in data exist but looking at outcomes other than the primary endpoint may be problematic, (10) particularly those that have not been pre-defined. (11) Lack of statistical power may lead to incorrect conclusions, but overpowered studies may invariably find a "statistically" significant finding with no clinical utility. (12)

\section{"Lack of statistical power may lead to incorrect conclusions, but overpowered studies may invariably find a "statistically" significant finding with no clinical utility. $(12)^{g !}$}

In planned comparisons, the data uniformly favors an exclusive human milk diet. The data support that EHMD is the standard for premature infants less than 1,250 grams. Data demonstrate a $77 \%$ reduction in NEC and meaningful reductions in many other common comorbidities (Sepsis, ROP, BPD, along with feeding intolerance, TPN, and length of stay). $(13,14)$

NEONATOLOGY TODAY is interested in publishing manuscripts from Neonatologists, Fellows, NNPs and those involved in caring for neonates on case studies, research results, hospital news, meeting announcements, and other pertinent topics.

Please submit your manuscript to: LomaLindaPublishingCompany@gmail.com 
What is more, the use of human milk has long been associated with other advantages, including reduced risk of diarrhea, leukemia, otitis media, viral respiratory tract disease, as well as certain metabolic disease processes, including type II diabetes and childhood obesity. (15-18) Neurodevelopmental studies and those that look at the quantification of white and grey brain matter suggest a definitive effect.

Additionally, the maternal benefits of breastfeeding cannot be ignored. Breastfeeding has been associated with decreased risk of type II diabetes, more rapid return to prepartum weight, decreased cardiovascular disease, modulation of hypertension, and reduced risk of metabolic syndrome. $(19,20)$ A reduced risk of breast and ovarian cancer has been demonstrated in those women who have breastfed. Even autoimmune disease processes may be mediated by breastfeeding. $(21,22)$

The cost of using bovine and other breastmilk substitutes reaches into the billions of dollars annually in the United States. (17, $23,24)$ Society is effectively subsidizing disparate outcomes in our most at-risk infants. Understanding the demographics of this situation is crucial. With the breastfeeding rates lowest in African American and Latinx families, these patterns worsen existing disparities. $(25,26)$ Inequities in access to resources to improve breastfeeding rates and lack of support in these communities for baby-friendly practices create boundaries that are often insurmountable for those most at risk.

\section{"The cost of using bovine and other breastmilk substitutes reaches into the billions of dollars annually in the United States. $(17,23,24)$ Society is effectively subsidizing disparate outcomes in our most at-risk infants."}

It is not appropriate to extrapolate data from pasteurized human milk studies to human milk sterilized by a different process.

The handling, the testing, and the processing of human milk are all critical to its makeup. Milk that is not processed appropriately is not equivalent to milk, that is. Protein, fat, vitamin, mineral, and micronutrient content and HMOs can be altered significantly if the integrity in processing is compromised. In particular, retort sterilization methods can produce results that may be suitable for canned tuna but not for a product as vulnerable as human milk. (27) Arguably, while a product made from the process may have a limitless shelf life, the process destroys the entire biologic value of human milk. There is a fundamental lack of data for retort sterilization. (28) How can this be acceptable? It is an experiment at best. Certain providers are selling an ill-defined product without relevant data -- A false narrative. At some point, it is crucial to demonstrate that human milk is still functionally human. Scientific rigor is meaningless if processing renders the product less than what it is claimed to be. (29)

No discussion about human donor milk is complete without at least a mention of safety. Although there is not a clear FDA qual- ification as to what constitutes and what is required for EHMD safety, it goes without saying that whether donor milk comes from a for- or not-for-profit model, the risks are still the same. Risks associated with pathogens and adulterants are not different between products made by milk banks of the two business models, as the only distinction between the two is merely their tax filing status. (30) Both for-profit and not-for-profit entities still have expenses and must pay their employees, provide safety screening, and ensure that adequate testing is in place to provide a safe final product. Both for-profit and not-for-profit milk banks are collecting human bodily fluid from a person outside of their direct proximity and control. The chance that a donor may inadvertently leave her child's daycare with milk belonging to someone else is not different between business models. The prescription drugs that donors are prescribed are not different, nor is the risk of exposure to harmful pathogens. Rigorous donor screening is not enough; the milk itself must be tested to ensure it is safe for the fragile infants it is destined to feed. (30-33)

The slightest error could be harmful or fatal for premature babies in Neonatal Intensive Care Units (NICUs). The possible long-term effects are myriad. These errors, when compounded, may mean the difference between an outcome that results in a baby that has a chance to reach a high potential and one that leads to developmental delay, multiple subspecialty appointments, increased risk of chronic disease, and growth failure. (34) Preemie parents, often feeling helpless and overwhelmed, place their faith and their child's life in the hands of physicians, nurses, and other hospital providers, doing everything humanly possible to ensure the best outcome for the baby. These clinicians must use the most scientifically sound information available to them. Knowledge of this information requires intense scrutiny of the literature and an understanding of how to interpret the findings for the best possible outcomes. For the healthcare team, every decision is made with the utmost care. Anecdotal reports have no place in this evaluation. Instead, evidence-based randomized control trials, other well-controlled data, and knowledge of the best information available must dictate best practice. That care often includes the use of human donor milk and human donor milk-derived products, including fortifiers. $(13,35)$

\section{"Anecdotal reports have no place in this evaluation. Instead, evidence-based randomized control trials, other well- controlled data, and knowledge of the best information available must dictate best practice. That care often includes the use of human donor milk and human donor milk-derived products, including fortifiers.} $(13,35)^{9 \prime}$

When analyzing studies, several questions must be asked:

1. Who is in the population? Preterm babies? Term babies? 
2. What is being compared? Human milk to cow milk? Traditional Pasteurization methods to those that are less well known or studied?

3. Is the term exclusive human milk diet truly exclusively human milk, or just human milk as a base with cow milk-based fortification?

4. Where is the study taking place? In the NICU? In the normal nursery? In a home environment?

5. Is this a randomized control study, a cohort study, a case report, or case series?

6. If this is a randomized control study, did a research board approve the study?

7. Is the study adequately powered? Is the sample size sufficient to make the comparison? Did patients drop out of the study? Were there enough patients at the end of the study to make an adequate comparison?

8. Do the statistics support a clinically relevant endpoint? Or is the endpoint trivial?

9. What are the implications for practice? Findings in term infants or more mature preterm infants may not be relevant to those at the margins of viability.

10. What is the feasibility of implementing these findings in other populations? Are the conclusions generalizable to broader clinical arenas?

11. What about cost? Will spending more upfront produce economies of scale, decrease morbidity or mortality, or decrease the length of hospitalization?

12. Are there long-term outcome data? Many therapies are too new to be looked at retrospectively, but where data exists, it should not be ignored.

\section{References:}

1. Abrams SA, Schanler RJ, Lee ML, Rechtman DJ. Greater mortality and morbidity in extremely preterm infants fed a diet containing cow milk protein products. Breastfeed Med. 2014;9(6):281-5. Epub 2014/05/29. doi: 10.1089/ bfm.2014.0024. PubMed PMID: 24867268; PubMed Central PMCID: PMCPMC4074755.

2. Assad M, Elliott MJ, Abraham JH. Decreased cost and improved feeding tolerance in VLBW infants fed an exclusive human milk diet. J Perinatol. 2016;36(3):216-20. Epub 2015/11/13. doi: 10.1038/jp.2015.168. PubMed PMID: 26562370

3. Cristofalo EA, Schanler RJ, Blanco CL, Sullivan S, Trawoeger $R$, Kiechl-Kohlendorfer $U$, et al. Randomized trial of exclusive human milk versus preterm formula diets in extremely premature infants. J Pediatr. 2013;163(6):15925 e1. Epub 2013/08/24. doi: 10.1016/j.jpeds.2013.07.011. PubMed PMID: 23968744.

4. Huston R, Lee M, Rider E, Stawarz M, Hedstrom D, Pence $M$, et al. Early fortification of enteral feedings for infants $<1250$ grams birth weight receiving a human milk diet including human milk based fortifier. J Neonatal Perinatal Med. 2020;13(2):215-21. Epub 2019/11/11. doi: 10.3233/ NPM-190300. PubMed PMID: 31707377; PubMed Central
PMCID: PMCPMC7369034.

5. Maffei $D$, Schanler RJ. Human milk is the feeding strategy to prevent necrotizing enterocolitis! Semin Perinatol. 2017;41(1):36-40. Epub 2016/11/12. doi: 10.1053/j.semperi.2016.09.016. PubMed PMID: 27836421.

6. Taggart Medo E. Response to Sakamoto et al. Breastfeed Med. 2014;9(3):168-9. Epub 2014/02/05. doi: 10.1089/ bfm.2013.0147. PubMed PMID: 24490933.

7. Segerstrom SC. Statistical Guideline No. 5. Include Results of a Power Analysis; if a Power Analysis Was Not Performed, Describe the Stopping Rule for Recruitment. Int J Behav Med. 2020;27(2):140-1. Epub 2020/03/07. doi: 10.1007/ s12529-020-09868-7. PubMed PMID: 32140932.

8. Ahmed Ali U, Ten Hove JR, Reiber BM, van der Sluis PC, Besselink MG. Sample size of surgical randomized controlled trials: a lack of improvement over time. J Surg Res. 2018;228:1-7. Epub 2018/06/17. doi: 10.1016/j. jss.2018.02.014. PubMed PMID: 29907196.

9. Cleophas $T J$, Zwinderman AH. Clinical trials are often false positive: a review of simple methods to control this problem. Curr Clin Pharmacol. 2006;1(1):1-4. Epub 2008/08/01. doi: 10.2174/157488406775268228. PubMed PMID: 18666373.

10. Brookes ST, Whitley E, Peters TJ, Mulheran PA, Egger M, Davey Smith G. Subgroup analyses in randomised controlled trials: quantifying the risks of false-positives and false-negatives. Health Technol Assess. 2001;5(33):1-56. Epub 2001/11/10. doi: 10.3310/hta5330. PubMed PMID: 11701102.

11. Rice TK, Schork NJ, Rao DC. Methods for handling multiple testing. Adv Genet. 2008;60:293-308. Epub 2008/03/25. doi: 10.1016/S0065-2660(07)00412-9. PubMed PMID: 18358325.

12. Gordi T, Khamis $H$. Simple solution to a common statistical problem: interpreting multiple tests. Clin Ther. 2004;26(5):780-6. Epub 2004/06/29. doi: 10.1016/s01492918(04)90078-1. PubMed PMID: 15220022.

13. Lucas A, Boscardin J, Abrams SA. Preterm Infants Fed Cow's Milk-Derived Fortifier Had Adverse Outcomes Despite a Base Diet of Only Mother's Own Milk. Breastfeed Med. 2020;15(5):297-303. Epub 2020/04/03. doi: 10.1089/ bfm.2019.0133. PubMed PMID: 32239968; PubMed Central PMCID: PMCPMC7232708.

14. Section on B. Breastfeeding and the use of human milk. Pediatrics. 2012;129(3):e827-41. Epub 2012/03/01. doi: 10.1542/peds.2011-3552. PubMed PMID: 22371471.

15. Huston RK, Markell AM, McCulley EA, Gardiner SK, Sweeney SL. Improving Growth for Infants $</=1250$ Grams Receiving an Exclusive Human Milk Diet. Nutr Clin Pract. 2018;33(5):671-8. Epub 2018/02/17. doi: 10.1002/ ncp.10054. PubMed PMID: 29451716.

16. Parker LA, Krueger C, Sullivan S, Kelechi T, Mueller M. Effect of breast milk on hospital costs and length of stay among very low-birth-weight infants in the NICU. Adv Neonatal Care. 2012;12(4):254-9. Epub 2012/08/07. doi: 10.1097/ ANC.0b013e318260921a. PubMed PMID: 22864006.

17. Bartick $M$, Reinhold $A$. The burden of suboptimal breastfeeding in the United States: a pediatric cost analysis. Pediatrics. 2010;125(5):e1048-56. Epub 2010/04/07. doi: 10.1542/ peds.2009-1616. PubMed PMID: 20368314.

18. Hornell A, Lagstrom H, Lande B, Thorsdottir I. Breastfeeding, introduction of other foods and effects on health: a sys- 
tematic literature review for the 5th Nordic Nutrition Recommendations. Food Nutr Res. 2013;57. Epub 2013/04/17. doi: 10.3402/fnr.v57i0.20823. PubMed PMID: 23589711; PubMed Central PMCID: PMCPMC3625706.

19. Schalla SC, Witcomb GL, Haycraft E. Body Shape and Weight Loss as Motivators for Breastfeeding Initiation and Continuation. Int J Environ Res Public Health. 2017;14(7). Epub 2017/07/12. doi: 10.3390/ijerph14070754. PubMed PMID: 28696385; PubMed Central PMCID: PMCPMC5551192.

20. Jacobson LT, Collins TC, Lucas M, Zackula R, Okut $H$, Nazir N, et al. Electronic Monitoring Of Mom's Schedule (eMOMS): Protocol for a feasibility randomized controlled trial to improve postpartum weight, blood sugars, and breastfeeding among high BMI women. Contemp Clin Trials Commun. 2020;18:100565. Epub 2020/04/30. doi: 10.1016/j. conctc. 2020.100565. PubMed PMID: 32346648; PubMed Central PMCID: PMCPMC7183152.

21. Babic A, Sasamoto N, Rosner BA, Tworoger SS, Jordan SJ, Risch $H A$, et al. Association Between Breastfeeding and Ovarian Cancer Risk. JAMA Oncol. 2020;6(6):e200421. Epub 2020/04/03. doi: 10.1001/jamaoncol.2020.0421. PubMed PMID: 32239218; PubMed Central PMCID: PMCPMC7118668.

22. Brinton LA, Potischman NA, Swanson CA, Schoenberg JB, Coates RJ, Gammon MD, et al. Breastfeeding and breast cancer risk. Cancer Causes Control. 1995;6(3):199-208. Epub 1995/05/01. doi: 10.1007/BF00051791. PubMed PMID: 7612799.

23. The economic value of breast-feeding. Food and Agriculture Organization of the the United Nations. FAO Food Nutr Pap. 1979;11:1-89. Epub 1979/01/01. PubMed PMID: 95882.

24. Choi YY, Ludwig A, Andreyeva T, Harris JL. Effects of United States WIC infant formula contracts on brand sales of infant formula and toddler milks. J Public Health Policy. 2020;41(3):303-20. Epub 2020/05/02. doi: 10.1057/s41271020-00228-z. PubMed PMID: 32355331.

25. Merewood A, Bugg K, Burnham L, Krane K, Nickel N, Broom $S$, et al. Addressing Racial Inequities in Breastfeeding in the Southern United States. Pediatrics. 2019;143(2). Epub 2019/01/20. doi: 10.1542/peds.2018-1897. PubMed PMID: 30659064

26. Spencer B, Wambach K, Domain EW. African American Women's Breastfeeding Experiences: Cultural, Personal, and Political Voices. Qual Health Res. 2015;25(7):97487. Epub 2014/10/08. doi: 10.1177/1049732314554097. PubMed PMID: 25288408.

27. Patel J, Parhi A, Al-Ghamdi S, Sonar CR, Mattinson DS, Tang J, et al. Stability of vitamin C, color, and garlic aroma of garlic mashed potatoes in polymer packages processed with microwave-assisted thermal sterilization technology. $J$ Food Sci. 2020;85(9):2843-51. Epub 2020/08/14. doi: 10.1111/1750-3841.15366. PubMed PMID: 32790195.

28. Lima HK, Wagner-Gillespie M, Perrin MT, Fogleman AD. Bacteria and Bioactivity in Holder Pasteurized and Shelf-Stable Human Milk Products. Curr Dev Nutr. 2017;1(8):e001438. Epub 2018/06/30. doi: 10.3945/cdn.117.001438. PubMed PMID: 29955718; PubMed Central PMCID: PMCPMC5998364.

29. Beliciu CM, Sauer A, Moraru Cl. The effect of commercial sterilization regimens on micellar casein concentrates. J Dairy Sci. 2012;95(10):5510-26. Epub 2012/08/21. doi: 10.3168/jds.2011-4875. PubMed PMID: 22901488.

30. Bloom BT. Safety of donor milk: a brief report. J Perinatol. 2016;36(5):392-3. Epub 2016/01/08. doi: 10.1038/ jp.2015.207. PubMed PMID: 26741570.

31. Davis NL, Corbett A, Kaullen J, Nelson JAE, Chasela CS, Sichali D, et al. Antiretroviral Drug Concentrations in Breastmilk, Maternal HIV Viral Load, and HIV Transmission to the Infant: Results From the BAN Study. J Acquir Immune Defic Syndr. 2019;80(4):467-73. Epub 2018/12/21. doi: 10.1097/ QAI.0000000000001941. PubMed PMID: 30570527; PubMed Central PMCID: PMCPMC6391211.

32. Updegrove $\mathrm{KH}$. Donor human milk banking: growth, challenges, and the role of HMBANA. Breastfeed Med. 2013;8(5):4357. Epub 2013/10/12. doi: 10.1089/bfm.2013.0079. PubMed PMID: 24112059.

33. Bertrand KA, Hanan NJ, Honerkamp-Smith G, Best BM, Chambers CD. Marijuana Use by Breastfeeding Mothers and Cannabinoid Concentrations in Breast Milk. Pediatrics. 2018;142(3). Epub 2018/08/29. doi: 10.1542/peds.20181076. PubMed PMID: 30150212; PubMed Central PMCID: PMCPMC6317767.

34. Fischer HR, Duncan SD. The business case for quality improvement. J Perinatol. 2020;40(6):972-9. Epub 2020/04/02. doi: 10.1038/s41372-020-0660-y. PubMed PMID: 32231258.

35. Hair $A B$, Peluso AM, Hawthorne KM, Perez J, Smith DP, Khan JY, et al. Beyond Necrotizing Enterocolitis Prevention: Improving Outcomes with an Exclusive Human Milk-Based Diet. Breastfeed Med. 2016;11(2):70-4. Epub 2016/01/21. doi: 10.1089/bfm.2015.0134. PubMed PMID: 26789484; PubMed Central PMCID: PMCPMC4782036.

Disclosures: There are no relevant disclosures identified.

NT

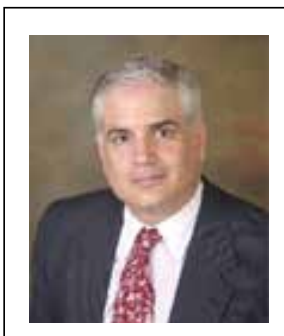

Mitchell Goldstein, MD, MBA, CML

Professor of Pediatrics

Loma Linda University School of Medicine

Division of Neonatology

Department of Pediatrics

Email: $\underline{\text { mgoldstein@llu.edu }}$ 